\title{
Clinical supervision in oncology: A narrative review
}

\author{
Natalie Hession, Andrea Habenicht \\ St Luke's Radiation Oncology Network, Dublin, Ireland
}

\begin{abstract}
The aim of this narrative literature review is to (a) review all empirical studies of clinical supervision (CS) in oncology and to (b) highlight some CS practice issues distinctive to cancer care. This review identified empirical studies related to qualified professionals and published in English since 1995 that connected to CS and oncology. A total of 15 studies were identified with the majority as small scale, exploratory and qualitative. Psychologists were predominantly utilised as facilitators of the CS with varied sample sizes ranging from 5-230 participants. The studies were grouped into three main categories; the impact of supervision on staff, impact of supervision on professional care/development and impact of supervision in existential exploration. This review highlighted the positive impact of CS while demonstrating the need for more methodologically sound programmes of research into CS in cancer settings so detailed models of effective supervision can develop and thereby inform practice.
\end{abstract}

Correspondence: Natalie Hession, Principal Counselling Psychologist, Head of Psycho-oncology, St Lukes Hospital, Dublin, Ireland. Tel: +353.1 .2453530$

E-mail: natalie.hession@slh.ie

Key words: Cancer, Supervision, Oncology, Reflect, Psychologist.

Contributions: $\mathrm{NH}$ is responsible for the conception and design of the work and the initial acquisition, analysis and interpretation of the data. $\mathrm{AH}$ was responsible for updating the manuscript including adding new articles and their subsequent analysis and interpretation to the document. Both authors edited and critically revised the completed piece.

Conflicts of interest: The authors declare no potential conflict of interest.

Funding: None.

Availability of data and materials: Not Applicable

Ethics approval and consent to participate: Not Applicable

Informed consent: Not Applicable

Received for publication: 1 November 2019.

Accepted for publication: 7 April 2020.

This work is licensed under a Creative Commons AttributionNonCommercial 4.0 International License (CC BY-NC 4.0).

C Copyright: the Author(s), 2020

Licensee PAGEPress, Italy

Health Psychology Research 2020; 8:8651

doi:10.4081/hpr:2020.8651

\section{Introduction}

This paper addresses clinical supervision (CS) in the psychosocial domain within cancer care. While CS is an integral aspect of mental health professionals training, it is not yet wellutilised within other healthcare professions (Edmonds et al., 2015). However, the application of CS within cancer care contexts is growing across disciplines due to the increase in psychosocial approaches to oncology that provide support and clinical care for the patient and then subsequently support the healthcare professional themselves. CS aims to develop and validate clinical practice, while exploring difficulties that arise in particular when working with co-morbid cancer and mental health presentations. CS provides a protected space to allow an educational and reflective process to occur via a typically more experienced 'supervisory' figure. This process allows individuals to reflect on the impact of clinical work on one's self, as practitioners are often faced with death and dying, one's own mortality may be questioned, or personal past experiences with death may evoke emotions in current work (Kangas-Niemia et al., 2018). Additionally, further challenges may arise in managing the intra- and inter- personal obstacles to provision of care that CS provides a platform to work through in a compassionate and reflective space. One of the most frequently provided definitions of CS is that of Inskipp and Proctor (2001): a working alliance between the supervisor and counsellor in which the counsellor can offer an account or recording of her work; reflect on it; receive feedback and where appropriate, guidance. The object of this alliance is to enable the counsellor to gain in ethical competence, confidence, compassion and creativity in order to give her best possible service to the client'. (p.1)

Within the working alliance, which is a central tenet in this definition, the work is reflected upon and feedback is provided in the interest of the professional development of the supervisee and patient welfare. This 'guidance' element provides a platform for education/professional development, while the use of 'compassionate' language is appropriate within the context of oncology. Aligned with this definition are the three functions of supervision (formative, normative and restorative) offered by Proctor (1987) which have been widely drawn upon as a framework in practice in counselling, therapy and nursing supervision. It is a particularly useful framework for considering the purpose of CS in cancer care as it emphasises the beneficial nature of CS on selfcare, while referencing professional standards and ethical considerations common place in oncology and emphasising skills and professional identity development which is important whilst working in large multi-disciplinary teams (MDT) in cancer care.

CS can help healthcare professionals to be better equipped to handle complex demands of working in oncology (Mackereth, Parkin, Donald, \& Antcliffe, 2010). Regardless of the setting, CS seeks to develop enhanced staff competence via responsive reflection, informed intervention and the reinforcement of the fact that the self, as presented in professional interaction, is dependent to a large extent on past experiences, the presence of others and 
overriding cultural context (Scaife, 2019). Therefore, an integral aspect of CS is exploring of the supervisors, supervisees and patients' perceptions of cultural identities, values and beliefs and how these both simultaneously affect, and are affected by engaging in work and how they shift over time. Through attention to important process issues CS, often provided by a psychologist, can help the cancer care professional address critical elements of their involvement in therapeutic relationships. Jones (2003) suggested that CS provides an environment in which to consider alternative future strategies to revise care management in a way that focuses interventions and enables appropriate disengagement to better equip healthcare professionals to handle the complex demands of working in oncology.

Increasingly, health professionals working in oncology are required to offer psychological support to patients in addition to clinical duties (Absolum et al., 2011). This can be emotionally demanding and without adequate training/support, staff may experience negative consequences. However, competing clinical demands may limit these healthcare professionals' abilities to provide psychosocial care suggesting the need to engage in CS with those individuals who have flexibility within their roles (Turner et al, 2018). In the UK and Ireland, counselling/clinical psychologists working in cancer care have established clinical supervision groups to support and guide health professionals in the holistic care of patients. As CS is becoming more established in oncology (Mackereth et al., 2010) the measurement of its effectiveness is an important challenge. Even though evaluation is difficult, proving its effectiveness is critical for sustainable implementation (Cummins, 2009), especially given the level of burnout/stress amongst healthcare professionals working in oncology (Brown, \& Bylund, 2008).

This paper will focus on the empirical research papers of CS in oncology regardless of the study design and operationalisation of the term. The aim of this narrative literature review is to review all empirical studies of CS in oncology and to highlight some CS practice issues that are pertinent to the psychologists providing CS and distinctive to the various healthcare professionals that use CS in cancer care. [Note: While CS can be facilitated by various healthcare professionals in the studies reviewed it was predominantly psychologists. Therefore, facilitators will be referred to as psychologists throughout the remainder of the review.] Additionally, this paper will identify issues that would benefit from additional research in the future. As CS requires both fiscal and human resources to be sustained, evidence from this review could be used to facilitate the adoption of CS.

\section{Method of literature review}

\section{Search strategy}

A keyword search included using the title 'oncology' or 'cancer care' and subject 'supervision' or title 'oncology' or 'cancer care' and title 'reflect*' was used. This was followed by a MeSH search included MeSH headings (mm "clinical supervision") and (mm "oncology"). Two overall strategies were used to search for empirical research papers on CS in oncology: (i) a combination of 'brief' and 'building block' search strategies (searching databases); and (ii) a citation pearl growing strategy (reviewing reference lists; Harter, 1986). The combined 'brief' and 'building block' search strategy was used to identify references in the following databases: Science Citation Index, CINAHL, MEDLINE, PubMed, PsycINFO and PsycARTICLES. The searches identified 38 articles. A total of 14 studies remained when the search was narrowed to articles that were published in a peer-reviewed scientific journal, were empirical in nature, published in English, related to trained/qualified healthcare professionals and were published between January 1994 and May 2019. There were no other restrictions in terms of setting, clinical speciality or whether CS was undertaken in a group or individual basis.

\section{Data analysis}

This paper uses a narrative review which aims to report the findings in a condensed format that provides the reader with a comprehensive overview and summarises the contents of each article (Green, Johnson, \& Adams, 2006) with the aim of presenting a broad perspective on the topic and bringing oncology practitioners up to date.

Articles using qualitative data were scored according to the NICE methodology checklist (2012) for qualitative studies and were rated independently by two researchers and disagreements discussed. The studies were assessed as to whether they fulfilled the three normative, formative and restorative functions of CS (Proctor, 1987). Articles failing to meet quality standards for qualitative research were classified as 'report-anecdotal' articles. Quantitative articles were narrowed by including only intervention studies. As this paper is a narrative review it does not systematically critically appraise the given literature and therefore a PRISMA checklist is not relevant. However, all studies were evaluated using the AMSTER checklist (Shea et al., 2007).

The narrative review analysis involved summarising all papers (Supplementary Table S1) according to i) setting and participants, ii) aim, iii) description of CS which included length and frequency of sessions, information on supervisors, the author's relationship to the CS, iv) methodology, v) results and vi) study limitations including design and CS limitations. Emphases, strengths and weaknesses were noted between the 14 papers. Major trends and subsequently relationships were identified among the studies resulting in three main categories, under which the studies were grouped and appraised. Finally, limitations and practice implications were identified in the literature.

\section{Data information}

Three different types of research designs were included; quantitative, qualitative and mixed methods. Two studies were prepost evaluation and twelve were post-only evaluation studies. The majority of the reviewed studies were small scale, exploratory and qualitative. Five of the studies were based in Sweden, four in the UK, one in the US and four in Australia. The studies participants focused on a range of healthcare professionals working in cancer care, predominately nurses as well as social workers, psychologists, physiotherapists, complementary therapists, doctors and occupational therapists. Sample sizes varied and ranged from 5-230 participants. As is commonly the case, the majority of the CS facilitators in the reviewed studies were psychologists (e.g. Sackin, \& Salinsky, 2012; Salander, \& Sandström, 2014).

\section{Results}

The studies were grouped into three main categories which arose from the literature as outlined below.

\section{Impact of supervision on staff}

The majority of the studies within this review addressed the impact of CS on staff. Through focus groups, Joubert, Hocking and Hampson (2013) found that social workers identified the importance 
of CS in supporting them in their professional practice. CS showed to play a role in mitigating the impact of vicarious trauma that manifested as hyper-sensitivity to possible symptoms that may relate to a cancer diagnosis. The social workers identified that it was the guidance provided by CS to manage the emotional impact of the work, as well as managing organisational challenges within the MDT and wider healthcare system that helped. This finding reflects what is widely accepted as an important restorative function of CS (Hawkins, \& Shohet, 2012; Inskipp and Proctor, 1995). However, these findings are limited in their generalisability due to the lack of control for confounding variables, inadequate sample size and vague description of the method. Mackereth, and colleagues' (2010) explored the value of CS and the experiences of complementary therapists working in oncology. Similar to Joubert et al. (2013), the analysis highlighted how CS was a supportive resource in particular enabling discussion and sharing feelings related to work. Confidentiality and boundaries, perceiving the CS facilitator as experienced, and support from management to regularly attend were the components that contributed to the value of CS. Given the noted significance of an experienced CS facilitator (Herbert \& Caldwell, 2015; McVey \& Jones, 2012), Mackereth et al's (2010) study highlights the importance of continued professional development and possible 'supervision of supervision' for facilitators in cancer care.

Beyond the supportive value of CS, McVey and Jones (2012) qualitatively evaluated the feedback from five CS groups with twelve nurses and one occupational therapist working in cancer, renal and neurological services and highlighted some practical considerations of CS. These include the importance of CS group make-up; that it should comprise of a range of professional viewpoints and a skilled CS facilitator to encourage crossprofessional learning. This paper highlights the perceived vulnerability of those disclosing and feeling safe by having a protected space for CS. The importance of feeling safe is not exclusive to CS in oncology and is frequently referred to as a necessity in supervision (Weaks, 2002). Furthermore, this article suggested CS enabled individuals to develop professionally through the group's ideas as well as learning psychological skills and exploring subconscious processes which typically go unexplored. However, interviews were short, varying from 10-30 minutes and capturing any in depth exploration within 10 minutes is typically challenging. Additionally, it is difficult to ascertain which service findings related to. Similar to McVey and Jones (2012) exploration of subconscious processes, Edmonds et al (2015) case study research suggested that expert-led group CS can develop a heightened awareness of the groups' emotions, biases and countertransference. This study presented a case example from a CS group that has been ongoing for over a year with all members of a palliative care team interested in partaking. CS was regarded as an avenue to develop/enhance self-awareness which was respectively viewed as an important aspect of self-care for palliative care staff. The CS sessions revolved around receiving general support with distressing cases and generating alternative interventions, whereby each week one participant brings a case that evoked a strong emotional reaction. Edmonds et al., (2015) suggested that when these subconscious processes of personal emotions, past experiences, biases and countertransference go unexplored this can subsequently lead to substandard patient care.

In Udo, Danielson, Henoch and Melin-Johansson's (2013) mixed method study they describe work-related stress in the care of the severely ill and dying, following participation in an existential educational intervention and supervised discussion on these existential areas. Immediately following the intervention nurses felt hindered in their caring, however six months later modest but significant differences were found indicating a long-term decrease in feelings of work and workload related stress, and less disappointment with their work. These findings align with studies outside of oncology which highlight the influence of CS in reducing burnout (Wallbank \& Hatton, 2011). Udo et al.'s (2013) methodology lacks standardised and validated evaluation measures and uses a small sample size $(n=42)$ therefore lacking rigor and robustness. Although, the authors mention a theoretical framework for the intervention, there is little detail regarding the content in terms of contract or supervision experience limiting the generalisability to CS in oncology. However, this is the only study within this literature review that randomised participants to a control/intervention group.

Ödling, Danielson and Jansson (2001) analysed the recorded supervision sessions of 21 healthcare professionals in a surgical ward for women with breast cancer in order to describe the content of their case presentations at CS sessions. Content analysis on 38 CS sessions focused on difficult care situations which related to discomfort around their own/patient's heightened emotions, feelings of powerlessness, and reduced self-esteem of staff/patients. The study illuminates the risk of burnout among palliative staff and highlighted how CS sessions offered an opportunity to reflect on difficult care situations with the hope of ameliorating this. It should be noted that the researcher was also the supervisor which could have led to positive bias. Pålsson, Hallbert, Norberg, and Isovaara, (1994) found nurses described CS as providing a sense of relief, either in immediate connection with a demanding situation or during CS itself. CS enabled opportunities to receive collegial responses to difficult situations and also generated a deeper knowledge, increased self-confidence and sense of well-being through facilitators focusing on the nurses' experiences/feelings rather than being solely solution focused. Similar to both Mackereth et al. (2010) and Joubert et al. (2013), the authors observed CS allowed staff to unburden emotionally. Developing a sense of group safety was instrumental, whereby staff knew feelings could be discussed at a dedicated time. Conversely, if group relationships were experienced as unsafe, difficultly in sharing emotionally demanding situations arose.

\section{Impact of supervision on professional care and develop- ment}

Few studies have looked at the efficacy of CS in terms of outcomes for the patient despite Ellis and Ladany stating that this is the 'acid test' of supervision (1997; p.485). In this review, four studies explored the benefit of CS to patient care in a cancer context. However, none of these studies directly evaluated patient care and thus findings were based on supervisees' self-reports. Xavier, Shepherd and Goldstein (2007) carried out an intervention analysis on 20 psychologists and social workers in 13 different Australian cancer centres. Findings observed significant increases in confidence in areas covered by the educational components and CS such as addressing issues related to death and dying, as well as significant increases in managing a range of psychological difficulties. This study used video conferencing and found it was both feasible and acceptable. The study showed an indication that CS via videoconference/telephone had an impact on patient care, however, these results were based on non-validated pre/post measures of supervisees' self-reports. Practical considerations such as sound quality and technical expertise need to be considered when contemplating such a technological approach to CS. Furthermore, in a cross-sectional study in Australia McMillan et al. (2016) 
explored the role of clinical supervision in 230 Cancer nurse's confidence in providing psychosocial care to patients and their own levels of burnout. This study observed that nurses who received frequent supervision they perceived to be adequate for their needs reported significantly less burnout than those nurses receiving less frequent or less adequately perceived supervision. Similar to Xavier, Shepherd and Goldstein's findings (2007), the nurses' confidence in their ability to provide psychosocial care increased as frequency and perceived adequacy increased. In turn these reported confidence levels were associated with increased level of professional efficacy suggesting that CS can be professionally beneficial twofold- through reducing risk of burnout, and through increasing confidence to provide care subsequent efficacy in their work.

In contrast to the above study's quantitative focus on perceptions of CS, Puffett and Perkins (2017) examined healthcare assistants and registered nurses' experiences of engaging or declining individual/group CS in palliative care. The study observed that individual CS was useful for personal work-related issues/emotions whereas group CS was beneficial for team building, identifying areas for further training and subsequently changing daily practice. Moreover, the research explored how those that did not engage in CS often utilised informal team support in times requiring immediate support. Beyond this, participants suggested there was unknown surrounding what CS actually is-with some believing it to be akin to an appraisal/work review. However, once this "mystique" was overcome and participants engaged in CS it led to positive individual/team professional development. Similar to McVey and Jones' (2012) study participants identified that a supervisor should be trained/well-experienced in CS in order to create a safe environment but there was no consensus on discipline or managerial status. Yet, only two members in the non-engaging group were interviewed for this research limiting the results' representation.

Salander and Sandström's (2014) exploratory study on Balintinspired groups with oncology doctors examined how such reflective supervision groups can be structured and what type of clinical oncology cases doctors are concerned with. Balint groups are one of the earliest forms of CS for doctors and are still widely used (Sackin, \& Salinsky, 2012). The authors describe the reflective groups, led by a psychologist, in detail and analysed 63 CS cases over 7 years using grounded theory. Findings highlighted communication challenges in patient-doctor relationships, in organisational contexts and in relation to patient's relatives. As aforementioned, Joubert et al. (2013) explored the impact of CS on social workers. The participants identified the importance of CS in supporting social workers in their professional practice through focusing on practice with patients and their families and the linking of this to social work theories/frameworks. CS also supported social workers in managing caseloads and organisational challenges within MDT and the wider healthcare system. Similarly, Turner et al (2018) examined how health professionals who engaged in the PROMPT study found CS enabled a safe setting whereby they could support/assist their colleagues through transferring knowledge. Numerous trained healthcare professionals engaged in weekly hourlong CS sessions whereby they discussed cancer patients experiencing depressive symptoms with whom they were conducting brief psychosocial interventions. This study aimed to embed psychosocial care into routine clinical practice aligning with Absolum and colleagues' (2011) observation that healthcare professionals are often expected to incorporate psychological support into their practice. The CS was viewed as a space to develop collegiality, ongoing professional learning and enhance self-efficacy through vicarious experience, all while receiving peer support.
Therefore, the CS in this study lead to self-reported increased confidence when engaging with patients as well as enhanced capacity to reflect on personal insights about clinical encounters. However, while this study emphasised that CS was highly valued and feasible in busy clinical settings, it also stated that the CS was based on Proctors (1987) principles yet failed to give any detail on the CS process or how these functions were incorporated.

\section{Impact of supervision on existential explorations}

Professionals working with illness and dying can confront personal values and beliefs. In Frankl's terms (1959) the supervisor would act as an 'eye specialist' extending the visual field of the worker so that: “...a whole spectrum of meaning and values becomes conscious and visible" (p.174). Addressing this issue, Udo, Melin-Johansson and Danielson (2011) explored healthcare professional's existential issues when caring for cancer patients. This secondary analysis, of a previous qualitative study on supervision sessions, produced themes related to the staffs' feelings of powerlessness; identifying with patients and getting close or keeping one's distance. Findings also related to staff's perception of patients' feelings of despair and isolation. Overall it reports a gap in knowledge regarding how surgical staff may support patients' existential well-being and how they can deal with personal feelings of powerlessness and identification with patients. This points to a role for CS to help staff develop strategies to deal with existential issues. Unfortunately, this study provided no information on the experience or how the CS sessions were contracted. Similar to Udo et al. (2011), existential concerns arose from Jones's (2001) analysis on supervision sessions of 5 nurses. These concerns related to death, freedom, responsibility, isolation and meaninglessness. Other themes included empathic insufficiencies and attainments in the organisation of palliative care, biological determinants and compulsion to caregiving. Jones (2001) was the only study within this narrative review that supervision was on an individual basis (two studies did not specify) which explains the low sample.

As aforementioned, Udo and colleagues' (2011) mixed method study describes work-related stress in the care of the severely ill and dying following participation in an existential educational intervention and supervised discussion. Specific to existential issues, post intervention results showed that colleagues better understood patients' emotional and existential reactions, such as sorrow and thoughts of death, following CS discussion and reflection. Qualitative results at 6 months showed that feelings of improved decision making were found whereby the staff felt they could meet the needs of severely ill patients and act according to their own caring intentions instead of rushing between patients.

These studies highlight the need for CS as a vehicle for staff to grapple with their own existential issues and subsequently support patients through theirs. It offers staff a time to accommodate all aspects of their experiences and an opportunity to understand the complexities of working with serious illness and dying.

\section{Discussion}

Many of the reviewed exploratory studies had clear hypotheses but few defined constructs and often supervision content was vague (e.g. Puffett \& Perkins, 2017; Udo et al., 2013). Beyond the lack of consensus definition about CS from a consistent methodology standpoint, Puffett and Perkins' (2017) study also noted the ambiguity some supervisees associated with the term. The word supervision may be unconsciously associated with formal authority- 
"supervisors", and subsequently hinder uptake due to job appraisal misconceptions rather than $\mathrm{CS}$ as related to self-care and professional development. This review also highlighted that the measurement of the supervision process and its impact on patient care was at times evaluated using non-validated scales and evaluation was based on supervisees' self-reporting (not patients) satisfaction surveys (e.g. Ellis \& Ladany, 1997). The direct impact of CS on the patient is unknown and the value of CS was very much focused on the staff engaging in supervision. This was a limiting factor for all reviewed studies and is mirrored in the wider field of CS. Training for supervisors is valuable and necessary (Williams \& Webb, 1994), however, only two (McVey \& Jones, 2012; Puffet \& Perkins, 2017) out of 15 studies reported specific supervisor training (four studies suggested facilitators had CS “experience"). Due to scant details on what CS looked like (e.g. Mackereth et al., 2010; McVey \& Jones, 2012), it was difficult to ascertain whether there was evidence of all three of Inskipp and Proctor's (1995) stated functions of CS (restorative, normative and formative) within the studies. As the origins of CS were formative (Brunero \& SteinParbury, 2008), the results may serve to reinforce the stressful nature of working in oncology whilst omitting the normative and restorative aspects of CS. Scaife (2019) suggests that CS should encompass all functions but the restorative aspect is often predominant as CS provides a space to reflect, and beyond using this space as a channel for subconscious emotions, it allows exploration into how emotions may be utilised constructively to enhance clinical work. However, Turner et al. (2018) suggested that the group CS format allowed for the restorative function of CS through peer validation/support, as well as the formative and normative components amidst transferring of group knowledge and consistent approaches to patient care. Therefore, perhaps CS in a psychosocial setting can address all three (Proctor, 1987) functions in an integrated manner as they intersect in real-world engagement, yet the current studies surrounding CS in oncology have failed to address/measure this overlap.

Supervision, occurs with a variety of oncology healthcare professionals, has various definitions, functions and modes of delivery. This complexity means that research into supervisory practice presents methodological problems. The majority of the reviewed studies were post CS studies and exploratory in nature. Firstly, this may be due to the lack of well-validated and reliable measures of CS effectiveness and secondly the opportunistic nature of post evaluation research. In addition, the supervisor was often the researcher (e.g. Odling et al., 2001), possibly creating positive biases. This review aligns with Dillworth et al. (2014)'s call for a clearer understanding of CS processes as it demonstrates the need for more methodologically sound research into $\mathrm{CS}$ in cancer settings, so that detailed models of effective supervision can be developed and thereby inform practice. Future research should include validated measures to assess effectiveness; the use of pre/post interventions; randomised controls; the direct measure of CS effectiveness on patient care; larger participant numbers and the use of experienced and/or trained supervisors in order to ensure consistency of supervision quality and safety. At the same time there is the importance of empirically distinguishing what is quantitatively different about CS in oncology to other healthcare areas.

\section{Practice implications and future directions}

The healthcare service faces increasing pressure to streamline and improve patient care while retaining staff, so it is important to understand the role that CS plays in this process. In the UK, The Manual for Cancer Services 2008 (NCAT, 2010) states members of
MDTs who are trained to deliver level 2 psychological support should also receive regular CS. In an Irish context, this mirrors the National Cancer Strategy 2017-2026 (Department of Health, 2017) which recommends investment into the delivery of comprehensive psycho-oncology services, from diagnosis into the survivorship phase. Furthermore, given stress/burnout are among the top priorities selected by oncology nurses in Ireland (Murphy \& Cowman, 2006) it seems pertinent that the role of CS in oncology is further researched in the psycho-oncology field given that psychologists/psychiatrists most often facilitate.

Furthermore, this narrative review omitted studies that included unqualified or in-training healthcare practitioners (Kangas-Niemi, Manninen \& Mattsson, 2018). Future research may examine if differences occur as particularly in cancer care settings, there may be a distinction between the frequency and focus of CS required by those training versus those well-established (i.e. burnout, compassionate fatigue; Joubert et al., 2013). Furthermore, a limitation of the data analysis methodology is that a narrative review is not as rigorous as a meta-analysis and is more open to author bias. However, a narrative review was deemed appropriate in order to provide a comprehensive overview of the current literature pertaining to CS specifically in oncology settings, and to further inform practitioners, rather than systematically critically appraising available research. This review clearly highlighted the positive impact of staff's well-being and professional development and highlighted certain conditions supervisors need to consider/implement to bring about positive impacts. For example, the general process of CS specific to oncology needs more structure in order to be methodologically sound (Dilworth, 2014). For those facilitating CS it would be important to define boundaries and develop a supervision contract (Mackereth et al's, 2010) to allow the creation of specific guidelines about the process of CS and address any issues of group safety to reduce difficulty in sharing emotionally demanding stories (Pålsson et al., 1994).

However, in particular this review emphasised that supervisors in cancer care need to focus on patient/staff existential issues. Thus, allowing a space for healthcare professionals to explore existential issues and support patients when faced with similar concerns during their cancer journey. Although people respond differently to traumatic information when exposed to threats to their existence, existential issues are likely to arise and many patients with a cancer disease wish to discuss this (Strang, Strang, \& Ternestedt, 2001). Therefore, including an existential focus within CS is a way towards capturing part of the essence of oncology practice. Perhaps future research should also consider the role of 'supervision of supervision' in cancer care, in particular, when working with existential issues of the CS themselves.

\section{Conclusions}

This review study highlights specific points of interest for both professionals who participate in CS groups and psychologists who hope to supervise as well as (future) CS researchers. From this review, there are no definitive answers as to what is distinctive and necessary for supervision or what extra training supervisors need in cancer care. However, it is evident that CS can incorporate educational and normative practice into restorative reflection through enabling healthcare professionals working in oncology to discuss their own experiences with death and dying, or existential issues that may arise with patients. Due to increasing demands on healthcare professionals to provide both clinical care and 
psychological support to patients (Absolum et al., 2011), as well as recent strategies (Department of Health, 2017; NCAT, 2010) calling for investment in psycho-oncology services, it would appear it is time to focus future research on the distinctive aspects of CS in cancer care. Thus, it seems appropriate and timely for oncologyspecific CS policies to be introduced and a more definitive guideline created.

Cancer care is frequently a cradle of uncertainty and distress. The profound nature of the work repeatedly confronts cancer professionals with issues related to significant losses with recurrent and sometimes brutal challenges, in situations for which there are no simple solutions (Jones, 1998). The sustaining, nurturing and enabling aspects of CS can help healthcare professionals to sustain, nurture and enable others who are in need of care.

\section{References}

Absolom, K., Holch, P., Pini, S., Hill, K., Liu, A., Sharpe, M., ... \& NCRI COMPASS Supportive and Palliative Care Research Collaborative. (2011). The detection and management of emotional distress in cancer patients: The views of health $\square$ care professionals. Psycho $\square$ Oncology, 20(6), 601-608. doi.org/10.1002/pon.1916

Brown, R. F., \& Bylund, C. L. (2008). Communication skills training: describing a new conceptual model. Academic Medicine, 83(1), 37-44. doi:10.1097/ACM.0b013e31815c631e

Brunero, S., \& Stein-Parbury, J. (2008). The effectiveness of clinical supervision in nursing: an evidenced based literature review. Australian journal of advanced nursing, 25(3), 86-94.

Cummins, A. (2009). Clinical supervision: The way forward? A review of the literature. Nurse Education in Practice, 9(3), 215220. doi.org/10.1016/j.nepr.2008.10.009

Department of Health (2017). National Cancer Strategy 2017-2026. Retrieved from http://health.gov.ie/blog/publications/nationalcancer-strategy-2017-2026/.

Dilworth, S., Higgins, I., Parker, V., Kelly, B., \& Turner, J. (2014). Examining clinical supervision as a mechanism for changes in practice: a research protocol. Journal of advanced nursing, $70(2), 421-430$.

Edmonds, K. P., Yeung, H. N., Onderdonk, C., Mitchell, W., \& Thornberry, K. (2015). Clinical supervision in the palliative care team setting: a concrete approach to team wellness. Journal of palliative medicine, 18(3), 274-277. doi.org/10.1089/ jpm. 2014.0248

Ellis, M. V., \& Ladany, N. (1997). Inferences concerning supervisees and clients in clinical supervision: An integrative review.

Frankl, V. E. (1959). Man's search for meaning. Washington Square Press: New York.

Green, B. N., Johnson, C. D., \& Adams, A. (2006). Writing narrative literature reviews for peer-reviewed journals: secrets of the trade. Journal of chiropractic medicine, 5(3), 101-117. doi.org/10.1016/S0899-3467(07)60142-6

Harter, S. P. (1986). Online information retrieval: concepts, principles, and techniques. Academic Press, Inc.

Hawkins, P., \& Shohet, R. (2012). Supervision in the Helping Professions: An Individual, Group and Organisational Approach; Milton Keynes: Open University Press.

Herbert, J. T., \& Caldwell, T. A. (2015). Clinical supervision. Counseling theories and techniques for rehabilitation and mental health professionals, 2, 443-462.
Inskipp, F., \& Proctor, B. (1995). Art, Craft and Tasks of Counselling Supervision: Pt. 1 \& 2. Twickenham: Cascade Publications.

Inskipp, F., \& Proctor, B. (2001). The art, craft \& tasks of counselling supervision, part 2: Becoming a supervisor: professional development for counsellors, psychotherapists, supervisors and trainers. Twickenham: Cascade.

Jones, A. (2003). Clinical supervision in promoting a balanced delivery of palliative nursing care. Journal of Hospice \& Palliative Nursing, 5(3), 168-175.

Jones, A. (2001). Some experiences of professional practice and beneficial changes derived from clinical supervision by community Macmillan nurses. European Journal of Cancer Care, 10(1), 21-30. doi: 10.1046/j.1365-2354.2001.00209.x

Jones, A. (1998). Some reflections on clinical supervision: an existential $\square$ phenomenological paradigm. European Journal of Cancer Care, 7(1), 56-62. doi.org/10.1046/j.1365-2354.1998. 00069.x

Joubert, L., Hocking, A., \& Hampson, R. (2013). Social work in oncology-Managing vicarious trauma-The positive impact of professional supervision. Social Work in Health Care, 52(23), 296-310. doi:10.1080/00981389.2012.737902

Kangas-Niemi, A., Manninen, K., \& Mattsson, J. (2018). Facilitating affective elements in learning-In a palliative care context. Nurse education in practice, 33, 148-153. doi.org/ 10.1016/j.nepr.2018.09.007

Mackereth, P. A., Parkin, S., Donald, G., \& Antcliffe, N. (2010). Clinical supervision and complementary therapists: An exploration of the rewards and challenges of cancer care. Complementary therapies in clinical practice, 16(3), 143-148. doi: 10.1016/j.ctcp.2010.01.001

McVey, J., \& Jones, T. (2012). Assessing the value of facilitated reflective practice groups. CanCer nursing praCtiCe, 11(8). doi:10.7748/cnp2012.10.11.8.32.c9357

McMillan, K., Butow, P., Turner, J., Yates, P., White, K., Lambert, S., ... \& Lawsin, C. (2016). Burnout and the provision of psychosocial care amongst Australian cancer nurses. European Journal of Oncology Nursing, 22, 37-45. doi.org/10.1016/ j.ejon.2016.02.007

Murphy, A., \& Cowman, S. (2006). Research priorities of oncology nurses in the Republic of Ireland. Cancer nursing, 29(4), 283290.

National Cancer Action Team, (2010). Manual for Cancer Services 2008: Psychological Support Measures. NCAT: London.

NICE (2012). Methodology Checklist: Qualitative Studies. Retrieved from http://publications.nice.org.uk/the-guidelinesmanual-appendices-bi-pmg6b/appendix-h-methodology-checkli st-qualitative-studies on 15th November 2015.

Ödling, G., Danielson, E., \& Jansson, L. (2001). Caregivers' descriptions of patients with advanced breast cancer in connection with supervision sessions in a surgical ward. Cancer Nursing, 24(1), 28-34.

Pålsson, M. B., Hallbert, I. R., Norberg, A., \& Isovaara, S. (1994). Systematic clinical supervision and its effects for nurses handling demanding care situations. Interviews with Swedish district nurses and hospital nurses in cancer care. Cancer Nursing, 17(5), 385-394.

Proctor, B. (1987). Supervision: a co-operative exercise in accountability. In M. Marken and M. Payne (eds), Enabling and Ensuring: Supervision in Practice. Leicester: National Youth Bureau, Council for Education and Training in Youth and Community Work. Pp. 21-34.

Puffett, N., \& Perkins, P. (2017). What influences palliative care 
nurses in their choice to engage in or decline clinical supervision?. International journal of palliative nursing, 23(11), 524-533. doi.org/10.12968/ijpn.2017.23.11.524

Sackin, P., \& Salinsky, J. (2012). The Balint group as a form of supervision. In D. Owen \& R. Shohet (Eds.). Clinical Supervision in the Medical Profession: Structured Reflective Practice. McGraw-Hill Education: Maidenhead, UK.

Salander, P., \& Sandström, M. (2014). A Balint-inspired reflective forum in oncology for medical residents: Main themes during seven years. Patient education and counseling, 97(1), 47-51. doi:10.1016/j.pec.2014.06.008

Scaife, J. (2019). Supervision in clinical practice: A practitioner's guide. Routledge.

Shea, B. J., Grimshaw, J. M., Wells, G. A., Boers, M., Andersson, N., Hamel, C., Porter, A. C., Tugwell, P., Moher, D., Bouter, L. M. (2007). AMSTAR Checklist. Retrieved from http://amstar. ca/Amstar_Checklist.php on 7th November 2015.

Strang, S., Strang, P., \& Ternestedt, B. M. (2001). Existential support in brain tumour patients and their spouses. Supportive Care in Cancer, 9(8), 625-633. doi: 10.1007/s005200100258

Turner, J., Mackenzie, L., Kelly, B., Clarke, D., Yates, P., \& Aranda, S. (2018). Building psychosocial capacity through training of front-line health professionals to provide brief therapy: lessons learned from the PROMPT study. Supportive Care in Cancer, 26(4), 1105-1112. doi:10.1007/s00520-017-3929-8
Udo, C., Danielson, E., Henoch, I., \& Melin-Johansson, C. (2013). Surgical nurses' work-related stress when caring for severely ill and dying patients in cancer after participating in an educational intervention on existential issues. European Journal of Oncology Nursing, 17(5), 546-553. doi:10.1016/j. ejon.2013. 02.002

Udo, C., Melin-Johansson, C., \& Danielson, E. (2011). Existential issues among health care staff in surgical cancer carediscussions in supervision sessions. European Journal of Oncology Nursing, 15(5), 447-453. doi:10.1016/j.ejon. 2010. 11.010

Wallbank, S., \& Hatton, S. (2011). Reducing burnout and stress: the effectiveness of clinical supervision. Community Practitioner, 84(7), 31-36.

Weaks, D. (2002). Unlocking the secrets of 'good supervision': A phenomenological exploration of experienced counsellors' perceptions of good supervision. Counselling and Psychotherapy Research, 2(1), 33-39. doi:10.1080/147331402 12331384968

Williams, P. L., \& Webb, C. (1994). Clinical supervision skills: a Delphi and critical incident technique study. Medical Teacher, 16(2-3), 139-157. doi.org/10.3109/01421599409006725

Xavier, K., Shepherd, L., \& Goldstein, D. (2007). Clinical supervision and education via videoconference: a feasibility project. Journal of Telemedicine and Telecare, 13(4), 206-209. doi: 10.1258/1357633077809079 